\title{
Labels de la construction : quelle contribution possible au facteur 4 ?
}

Sustainable construction labels: Which contributions to the factor 4 objective?

Jonathan Villot, Natacha Gondran et Valérie Laforest

\section{(2) OpenEdition}

12 Journals

\section{Édition électronique}

URL : http://journals.openedition.org/developpementdurable/8834

DOI : 10.4000/developpementdurable.8834

ISSN : 1772-9971

Éditeur

Association DD\&T

\section{Référence électronique}

Jonathan Villot, Natacha Gondran et Valérie Laforest, « Labels de la construction : quelle contribution possible au facteur 4 ? », Développement durable et territoires [En ligne], Vol. 2, n 1 | Mars 2011, mis en ligne le 31 mars 2011, consulté le 10 décembre 2020. URL : http://journals.openedition.org/ developpementdurable/8834 ; DOI : https://doi.org/10.4000/developpementdurable.8834

Ce document a été généré automatiquement le 10 décembre 2020.

Développement Durable et Territoires est mis à disposition selon les termes de la licence Creative Commons Attribution - Pas d'Utilisation Commerciale 4.0 International. 


\section{Labels de la construction : quelle contribution possible au facteur 4 ?}

Sustainable construction labels: Which contributions to the factor 4 objective?

Jonathan Villot, Natacha Gondran et Valérie Laforest

1 Le facteur 4, concept ayant évolué au cours des décennies et des échelles d'étude, est une notion censée répondre aux enjeux du changement climatique et de l'épuisement des ressources énergétiques fossiles. Ciblant les émissions de gaz à effet de serre (GES) des pays dits "développés", il a été traduit pour les différents secteurs émetteurs par la définition d'objectifs appliqués aux consommations d'énergie. Les consommations d'énergie, quelle qu'elles soient, contribuent aux émissions de GES, et accentuent les dérèglements climatiques et la vulnérabilité des hommes et des écosystèmes. Le terme "facteur 4 " peut donc faire référence à deux ensembles distincts mais reliés entre eux : le facteur 4 climatique (objectif macro au niveau mondial) et le facteur 4 énergétique (objectif micro et cadre d'actions). L'application de ces concepts par les acteurs qu'ils soient politiques ou institutionnels a été fortement conditionnée par leurs objectifs, sensibilités et points de vue. Le secteur du bâtiment est l'un des premiers à avoir abordé ces concepts. La volonté de normalisation et valorisation des différentes démarches qui ont émergé a conduit à la mise en place des labels de la construction. Cet article vise, d'une part, à préciser les différentes définitions données à la notion de facteur 4 pour le bâtiment et, d'autre part, à essayer d'évaluer en quoi les différents labels contribuent, ou non, à l'atteinte du facteur 4 par ce secteur d'activité. Nous présentons ici les résultats d'une étude effectuée, début 2009, en collaboration avec l'Institut Français pour la Performance Énergétique du Bâtiment (IFPEB) qui a consisté à analyser 10 labels internationaux. L'ensemble des labels français à vocation nationale, publiés en janvier 2009, a été étudié ainsi que des labels étrangers et/ou à vocation internationale. 


\section{Du facteur 4 aux facteurs 4 : de quoi parle-t-on?}

\subsection{Facteur 4 : l'apparition d'un concept dans la littérature}

Le terme facteur 4 est introduit pour la première fois en 1997 dans un rapport au Club de Rome (von Weizsäcker et al., 1997). Ce document, par l'application de cinquante exemples concrets tente de démontrer la possibilité et l'opportunité de progrès que pourrait entraîner la notion de facteur 4. Le progrès, perçu comme synonyme d'amélioration a été porté depuis la fin du XIX ${ }^{\text {ème }}$ siècle par la notion de productivité du travail. Le taylorisme et le fordisme ciblaient l'augmentation du rendement et de la production par une organisation scientifique du travail (Taylor, 1911). Si ces méthodes étaient efficaces économiquement dans un monde où les ressources naturelles étaient considérées comme infinies et où les hommes étaient considérés pour leur seule capacité de production à court terme, elles se heurtent aujourd'hui au mur de la réalité environnementale et sociale. Dans ce réel, le progrès ne semble plus pouvoir être atteint uniquement par la productivité du travail. "L'utilisation de hauts quanta d'énergie a des effets aussi destructeurs pour la structure sociale que pour le milieu physique. Un tel emploi de l'énergie violant la société et détruisant la nature » (Illich, 1973).

L'approche «facteur 4 » propose une alternative à cette impasse en apportant des solutions concrètes à un développement dans lequel le progrès serait provoqué non plus par la productivité du travail mais par celle des ressources. Dans cette optique, le facteur 4 est défini par von Weizsäcker et al., comme "le coefficient permettant une multiplication par quatre de la productivité des richesses, c'est-à-dire une utilisation quatre fois plus importante de la même quantité de ressources ». Ainsi, «si la productivité des ressources était multipliée par quatre, le monde serait deux fois plus prospère que maintenant, tout en diminuant de moitiéla pression exercée par l'humanité sur son environnement ». Autrement dit, l'humanité pourrait prétendre à «deux fois plus de bien-être tout en consommant deux fois moins de ressources". Cette définition du facteur 4 montre un lien étroit entre productivité des ressources, richesses, bien-être, et pression sur l'environnement. L'approche « facteur 4 » de cette fin $\mathrm{du} \mathrm{XX}^{\mathrm{e}}$ siècle dévoile par son caractère englobant des répercussions impactant aussi bien les sphères économiques ( $\mathrm{x} 4$ des richesses), sociales (le bien-être), qu'environnementales (diminution par 2 de la pression sur l'environnement) (figure 1). 


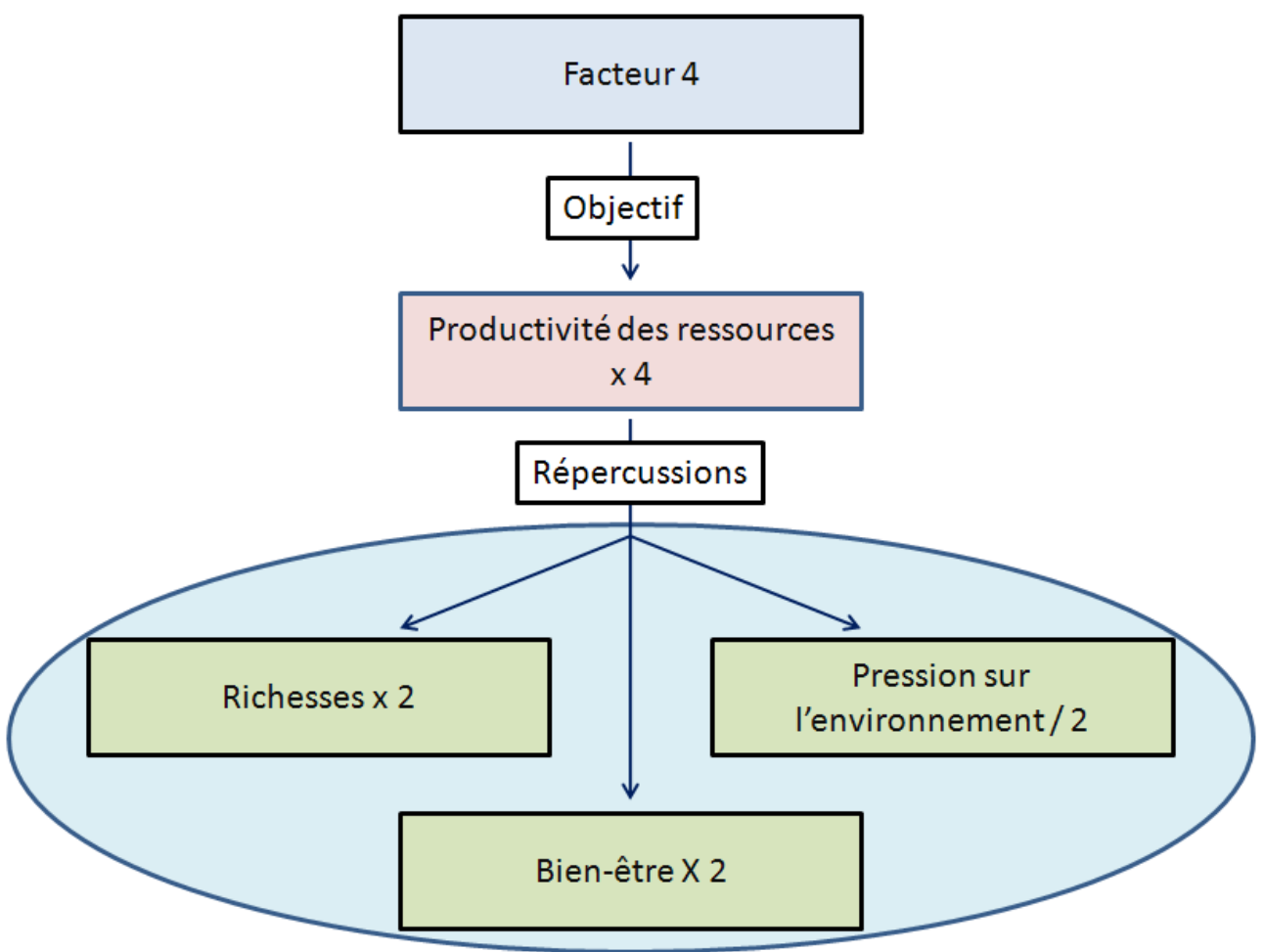

Source : Auteurs

4 Au début des années 2000, sous le poids des préoccupations sociétales et climatiques, le concept initialement large va se modifier pour se focaliser sur les émissions de gaz à effet de serre (GES). Il va alors être approprié par différents acteurs afin de représenter l'effort de diminution (d'un facteur 4) à mener par les pays industrialisés dont la France, en vue de ramener, à l'horizon 2050, les émissions de gaz à effet de serre (GES) à un niveau jugé acceptable et équitable sur le plan mondial.

\subsection{L'adaptation du facteur 4 aux questions climatiques.}

Qu'est ce que le facteur 4 aujourd'hui en France ? À quoi fait-il référence ? Et pourquoi est-il associé aux changements climatiques? Les rapports successifs du GIEC (Groupe Intergouvernemental d'Experts sur l'évolution du Climat), entre 1990 et 2007, ont mis en évidence le fait que des changements climatiques sont en cours et que la responsabilité de ces changements peut être attribuée aux activités humaines. Dans son quatrième rapport, le GIEC envisageait, selon différents scénarios d'évolutions des émissions de GES, des variations de température moyenne du globe comprises entre 1,1 et $6,4^{\circ} \mathrm{C}$ (GIEC, 2007). Par convention, le dioxyde de carbone $\left(\mathrm{CO}_{2}\right)$ est utilisé pour représenter les concentrations atmosphériques (en ppm) (stock) car c'est le principal contributeur au renforcement de l'effet de serre. En ce qui concerne les flux de gaz à effet de serre émis par l'homme, les émissions exprimées en $\mathrm{t}_{\mathrm{eq}} \mathrm{CO}_{2}$ représentent les gaz à effet de serre, notamment ceux concernés par le protocole de Kyoto et celui de Montréal. Ainsi, sous la "bannière $\mathrm{du} \mathrm{CO}_{2}$ ", on retrouve une équivalence pour différents GES: dioxyde de carbone $\left(\mathrm{CO}_{2}\right)$, méthane $\left(\mathrm{CH}_{4}\right)$, oxyde nitreux $\left(\mathrm{N}_{2} \mathrm{O}\right)$, hydrofluorocarbones (HFCs), hydrocarbure perfluorés (PFCs) et hexafluorure de soufre (SF6). Cette équivalence est 
établie par le "pouvoir de réchauffement global » (PRG) de chaque gaz qui représente sa contribution à l'effet de serre, relativement à l'effet généré par le $\mathrm{CO}_{2}$ sur une durée, conventionnellement choisie, de 100 ans. Depuis la période préindustrielle, les concentrations en $\mathrm{CO}_{2}$ ont largement augmenté. En 2005, la concentration atmosphérique a atteint $379 \mathrm{ppm}$ soit $35 \%$ de plus que les valeurs préindustrielles (280 ppm), conséquence d'une augmentation des flux de plus de 70\% entre 1970 et 2005 (GIEC, 2007).

Bien que le GIEC ne fasse pas explicitement référence au facteur 4, cet objectif est basé sur les scénarios de ce groupe d'experts. En effet, selon le GIEC, pour limiter au maximum la vulnérabilité et faciliter l'adaptation des écosystèmes et population actuels aux changements climatiques, la hausse moyenne des températures sur le globe ne devrait pas excéder $2^{\circ} \mathrm{C}$. Cela correspond pour le scénario le plus optimiste à une concentration atmosphérique maximale de $\mathrm{CO}_{2}$ en 2050 de 450 ppm. En 2050, le flux d'émissions devrait donc avoisiner les $4 \mathrm{Gt}$ de carbone par an au niveau planétaire (GIEC, 2001). En posant l'hypothèse d'une population mondiale à 6,7 milliards (Population Reference Bureau, 2008), cela revient à attribuer par habitant une possibilité d'émission limitée à 0,6 teqC/ an contre 2,4 teqC/an actuellement pour un Français moyen. Cela représente donc une division par 4 des émissions nationales françaises par habitant, par rapport à 1990 (GT Facteur 4, 2006). Le facteur 4 est donc présenté ici comme la division par 4 des émissions françaises nécessaires à la stabilité mondiale des flux, des stocks et de la température (respectivement à $4 \mathrm{Gt} \mathrm{C}, 450 \mathrm{ppm}$ et $2^{\circ} \mathrm{C}$ ) (figure 2).

7 Tenir de tels objectifs implique la mise en place de plans d'actions au niveau mondial comme national. La France, en tant que pays de l'annexe I (Nations Unies, 1998) du protocole de Kyoto est tenue à des objectifs de stabilisation des émissions de GES à court terme. Cependant, l'urgence de la situation lui impose d'agir par des politiques volontaristes et chiffrées sur ses secteurs les plus émetteurs de GES. 
Figure 2. Le facteur 4 climatique et ses répercussions directes

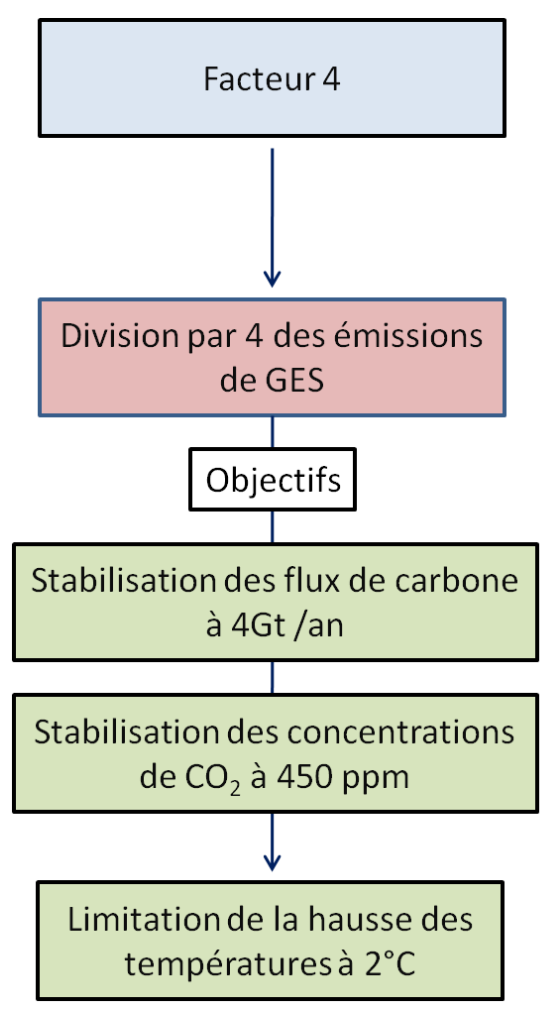

Source : Auteurs

\subsection{L'apparition de l'objectif dans les politiques françaises.}

8 En France, l'expression «facteur 4 » entre dans le champ de la décision politique le 19 février 2003 lors de l'allocution d'ouverture de la $20^{\mathrm{e}}$ session du GIEC par le premier ministre de l'époque Jean Pierre Raffarin (Tuddenham, 2006). La loi n ${ }^{\circ}$ 2005-781 du 13 juillet 2005 de Programme fixant les Orientations de la Politique Énergétique (Loi POPE) va officialiser l'engagement pris par la France (Loi POPE, 2005). Cette loi, par son article 2, « vise une diminution de $3 \%$ par an en moyenne des émissions de gaz à effet de serre de la France ... et soutient la définition d'un objectif de division par deux des émissions mondiales de gaz à effet de serre d'ici à 2050, ce qui nécessite, compte tenu des différences de consommation entre pays, une division par quatre ou cinq de ces émissions pour les pays développés ».

9 La publication de cette loi n'est que l'aspect législatif d'une politique de réduction des émissions de GES commencée à la signature du protocole de Kyoto et affirmée par les plans nationaux successifs. De fait du PNLCC (Plan National de Lutte contre les Changements Climatiques) en 2000, au Grenelle de l'environnement (2008) en passant par le Plan Climat (2004), les objectifs à court comme à long termes se sont orientés vers le facteur 4 (figure 3). 
Figure 3. Estimation des objectifs des différents plans nationaux



Source : Grenelle Groupe 1, 2008a

10 Malgré tout, l'affichage d'objectifs globaux théoriques ne peut conduire seul à un résultat. Des actions fortes et ciblées doivent être menées sur l'ensemble des secteurs émetteurs de GES au niveau national. Les plans énoncés ci-dessus s'attardent à définir des plans d'action par secteur émetteur en ciblant particulièrement ceux dont le poids ou la «facilité » en font des candidats de choix pour atteindre le facteur 4. Un des secteurs les plus emblématiques est le secteur du bâtiment qui représente plus d'un cinquième des émissions nationales et dont les marges de manœuvre (rénovation de bâtiments, par exemple) présentent la double particularité de générer de l'activité économique et d'améliorer le confort et la situation sociale des habitants.

\subsection{L'application du facteur 4 climatique aux bâtiments}

11 Pourquoi le bâtiment ? En quoi ce secteur d'activité plus que tout autre a-t-il la capacité de répondre (en partie) à l'objectif du facteur 4 climatique?

L'atténuation des émissions de GES au niveau mondial ne peut passer que par des actions ciblées sur l'ensemble des secteurs émetteurs. Deux secteurs ressortent cependant du lot de part l'évolution de leurs émissions depuis les années 1990: le transport et le résidentiel-tertiaire. En France, le transport était en 2007 le plus gros émetteur de GES (eq $\mathrm{CO}_{2}$ ) contribuant à $30 \%$ des émissions nationales et accusant une augmentation de $17,1 \%$ par rapport à 1990. Le bâtiment (résidentiel-tertiaire) est quant à lui, selon que l'on observe les émissions de $\mathrm{CO}_{2}$ ou les GES dans leur ensemble positionné à la seconde ou quatrième place des secteurs les plus émetteurs (figure 4). Tout comme le transport, le bâtiment connaît une augmentation de ses émissions depuis 20 ans $(+6 \%$ entre 1990 et 2007). 
Figure 4. Émissions de GES en France par secteur d'activité

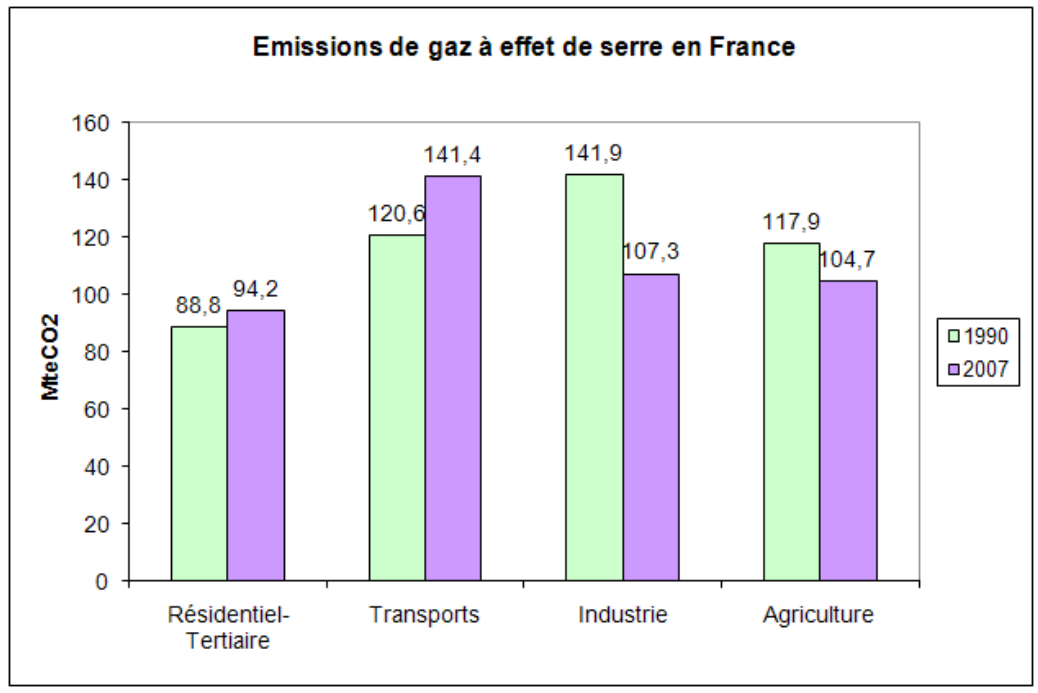

Source : MIES, 2008

Le bâtiment se démarque cependant des autres secteurs et surtout du transport par son fort potentiel économique de réduction d'émissions. En effet, les études prospectives du GIEC à l'horizon 2030 montrent que le potentiel d'atténuation économique pour le secteur du bâtiment serait compris entre 5,3 et $6,7 \mathrm{Gt}$ eq $-\mathrm{CO}_{2} /$ an pour un investissement inférieur à 100 \$ E-U/t eq - $\mathrm{CO}_{2}$ (figure 5) (GIEC, 2007).

Figure 5. Potentiel économique d'atténuation mondial en 2030 selon les études ascendantes du GIEC

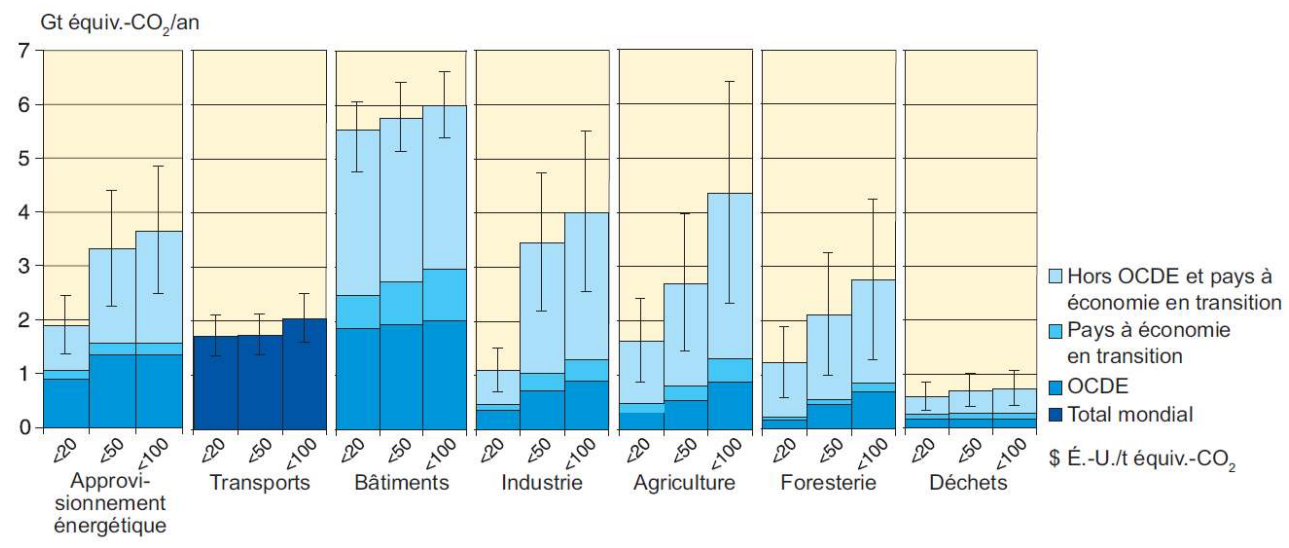

Source : GIEC, 2007

Ce potentiel de réduction des émissions de gaz à effet de serre dans le secteur du bâtiment est lié à plusieurs facteurs. Par exemple, de fortes réductions des consommations énergétiques, et des émissions de gaz à effet de serre associées, peuvent être espérées d'une amélioration technique des bâtiments (isolation de l'enveloppe, optimisation des usages, etc.). Ces améliorations, ne nécessitent pas une remise en cause profonde des comportements et organisations sociales et pourraient, par la mise en place d'un programme ambitieux de la qualité et des performances du bâti, générer de l'activité économique (Grenelle Groupe 1, 2008b). 
De plus, et contrairement à des secteurs tels que l'agriculture (émetteur de méthane et protoxyde d'azote) et l'industrie (émettrice de divers gaz à effet de serre dont certains ont des pouvoirs de réchauffement global très élevés), les principales sources de gaz à effet de serre du secteur du bâtiment sont liées aux consommations énergétiques (et, dans une moindre mesure, aux fuites d'hydro-fluoro-carbones présentes dans les installations de réfrigération et autres pompes à chaleur).

En France, le résidentiel tertiaire est ainsi le premier consommateur d'énergie avec plus de 63,3 millions de tonnes d'équivalent pétrole soit plus de $40 \%$ de l'énergie finale (MEEDDEM, 2007). Contrairement au transport consommateur d'une énergie unique (le pétrole sous la forme des supercarburants et du diesel), le bâtiment possède une mixité énergétique importante (figure 6). Cette mixité augmente les possibilités d'atténuation d'un facteur 4 des émissions dues à la consommation d'énergie dans le bâtiment en permettant le remplacement des énergies les plus émettrices (Fioul par exemple). Ainsi, contrairement aux transports, le bâtiment présente deux voies de réduction des émissions de GES :

- La réduction des consommations d'énergie

- Une modification du mix énergétique utilisé

Figure 6. Répartition des consommations du secteur résidentiel-tertiaire par type d'énergie

Résidentiel : 46,7 Mtep



mCharbon = Fioul $=\mathrm{GPL}=\mathrm{Gaz}$ Electricité $=$ Bois
Tertiaire : 22,4 Mtep

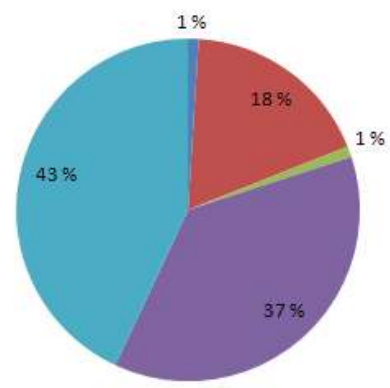

Source : MEEDDEM, 2007

17 Ainsi, en appliquant la définition du facteur 4 climatique proposé au niveau macroéconomique, l'atteinte de cet objectif dans le domaine du bâtiment en France consisterait à passer en moyenne d'une émission de $35 \mathrm{kgCO}_{2} / \mathrm{m}^{2} /$ an (Moyenne d'émission des logements français pour le chauffage et l'eau chaude sanitaire (Grenelle Groupe 1, 2008b) à moins de $9 \mathrm{kgCO}_{2} / \mathrm{m}^{2} / \mathrm{an}$. Cependant, au niveau micro-économique, les acteurs s'approprient plus facilement un objectif de réduction de leurs consommations énergétique (consommation directement reliée aux aspects économiques) qu'un objectif d'émissions de GES plus intangible. Le facteur 4 s'est donc traduit, dans les discussions du groupe de travail du grenelle consacré au bâtiment par la diminution de la consommation d'énergie de $240 \mathrm{kWh}_{\mathrm{ep}} / \mathrm{m}^{2} /$ an (Moyenne de consommation des logements français pour le chauffage et l'eau chaude sanitaire (Grenelle Groupe 1, 2008b) à $60 \mathrm{kWh}_{\mathrm{ep}} / \mathrm{m}^{2} / \mathrm{an}$. Ainsi, au-delà, d'une diminution des émissions, "le "facteur 4" signifie modifier sensiblement nos modes de consommation et de production, tout en augmentant fortement notre efficacité 
énergétique... » (CIADD, 2008). Le facteur 4 « énergétique » apparait donc ici comme une notion plus concrète pour les acteurs de terrain.

\section{Le facteur 4 climatique VS énergétique}

Bien que l'objectif du facteur 4 climatique soit régulièrement affiché en tant qu'objectif prioritaire, comme en témoignent les plans successifs (PNLCC, Plan Climat, Grenelle), les textes de lois européens et nationaux ne fixent que des objectifs globaux de diminution de GES. Aucun objectif n'est sectorisé en France pour les émissions de GES. Seul l'objectif énergétique est mentionné dans les engagements sectoriels. Deux niveaux se distinguent alors :

- Un objectif national, au niveau macro-économique : le facteur 4 climatique avec un objectif en $\mathrm{t}_{\mathrm{eq}} \mathrm{CO}_{2}$ ou $\mathrm{CO}_{2}$;

- Des objectifs sectoriels, au niveau micro-économique : le facteur 4 énergétique avec un objectif selon les secteurs en tep, ou $\mathrm{kWh}_{\mathrm{ep}}$.

19 Les objectifs sectoriels, c'est-à-dire le facteur 4 énergétique, s'appliquent notamment au bâtiment. Ainsi, pour 2020 l'objectif fixé par le Grenelle de l'environnement est de $-40 \%$ de consommation d'énergie par rapport à l'actuel sur le parc national de logement. Ces objectifs passent principalement par l'accentuation de la réglementation thermique qui périodiquement (tous les cinq ans) se renforce de 15\%. De ce fait, en 2012, les bâtiments neufs devront respecter le niveau bâtiment basse consommation (BBC) soit une consommation de $50 \mathrm{kWh}_{\mathrm{ep}} / \mathrm{m}^{2} /$ an (Grenelle Groupe 1, 2008b). Pour le bâtiment ancien, l'objectif pour 2012 est en moyenne de $-12 \%$ par rapport à l'actuel. Cet objectif reste fortement influencé par le taux de rénovation qui pour s'orienter vers le facteur 4 doit maintenir un taux de 400000 rénovations par an (Sidler, 2003). Objectif qui, sans obligation de travaux notamment dans le secteur privé, reste conditionné à une vague de démarches volontaires de la part des propriétaires.

Le fait de décliner par secteur le facteur 4 énergétique peut s'expliquer par sa capacité à répercuter des effets bénéfiques au-delà des simples émissions de $\mathrm{CO}_{2}$. En effet, il permet de répondre aux objectifs de la directive européenne et de la loi POPE. Objectifs qui dépassent les aspects climatiques. De fait, l'efficacité énergétique par la baisse de la consommation garantit une plus grande maitrise de l'énergie (Loi POPE, 2005) permettant à la France de :

contribuer à l'indépendance énergétique nationale et garantir la sécurité d'approvisionnement ;

21 1- assurer un prix compétitif de l'énergie ;

2- préserver la santé humaine et l'environnement, en particulier en luttant contre l'aggravation de l'effet de serre ;

3- garantir la cohésion sociale et territoriale en assurant l'accès de tous à l'énergie.

22 Par ces objectifs, la loi POPE se rapproche de la définition donnée au facteur 4 en 1997, soit un concept touchant les sphères économiques, sociales et environnementales. Elle associe ainsi à l'objectif climatique (facteur 4 climatique) des objectifs énergétiques directement connectés aux, enjeux économiques, tout en affichant une volonté de cohésion sociale et territoriale (figure 7). 
Figure7. Le facteur 4 énergétique et ses possibles répercussions

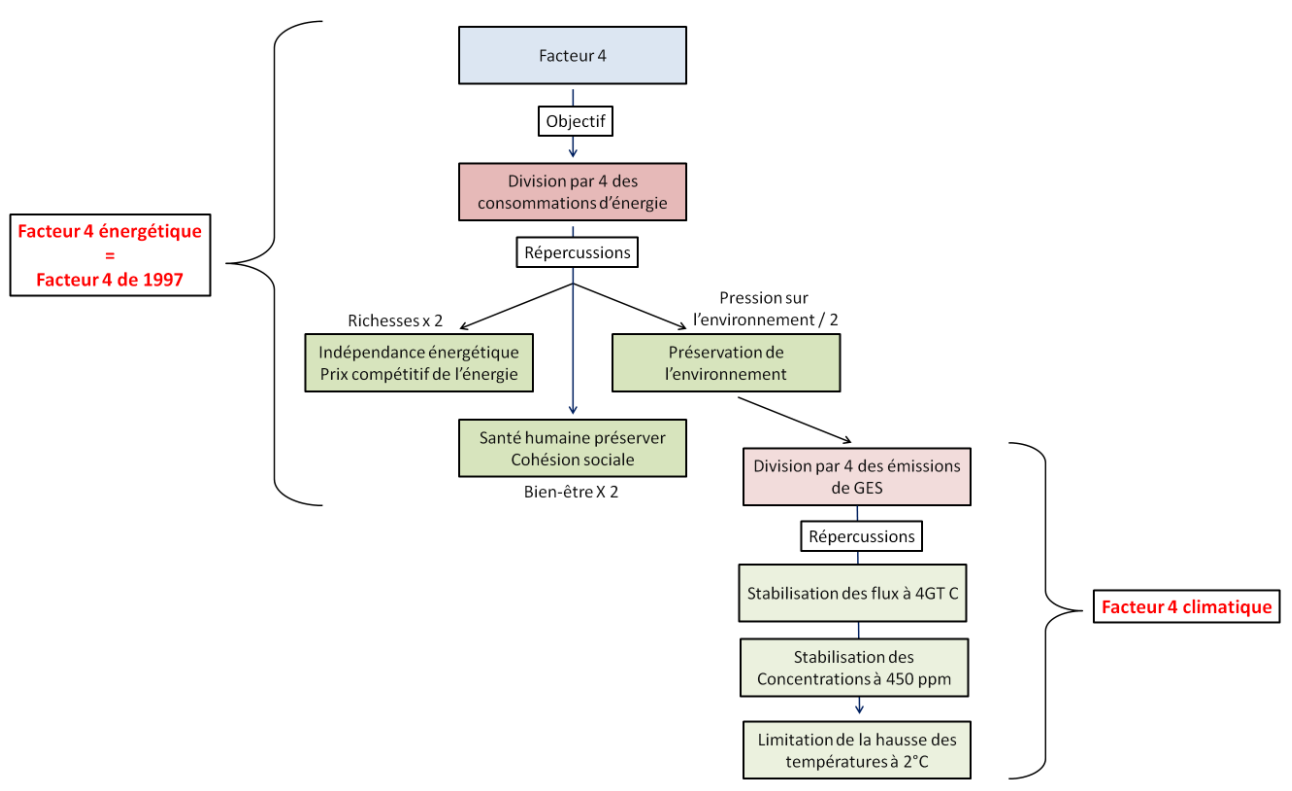

Source : Auteurs

Maîtriser l'énergie en vue d'atteindre les objectifs du facteur 4 ne peut cependant se limiter à la seule efficacité des techniques et technologies. Partant du principe qu'il existe "une alternative crédible à l'augmentation infinie de nos consommations d'énergie", l'association Négawatt par sa démarche apporte une réponse (Association négaWatt, 2006). En effet, la démarche NégaWatt propose une approche en trois temps permettant de réduire les consommations d'énergie: le premier temps consiste à supprimer les divers gaspillages énergétiques au niveau de nos comportements et organisations (étape de "sobriété »). La deuxième étape consiste à optimiser l'utilisation des ressources pour répondre à un besoin donné en utilisant les techniques et méthodes les plus efficaces (" efficacité énergétique »). Enfin, le dernier temps de la démarche consiste à privilégier l'utilisation d'énergies renouvelables. Bien entendu la sobriété n'équivaut pas à un rationnement mais doit être envisagée comme une réponse à la frénésie de nos consommations. Ainsi «c'est en changeant le regard que nous portons sur nos consommations d'énergies ", et en développant le marché des négawatts (watt non utilisé), que les facteurs 4 énergétique et climatique seront atteignables.

Il est indéniable même au travers de la démarche négawatt que l'interconnexion et les rétroactions entre les objectifs du facteur 4 climatique, énergétique, les enjeux économiques et de la cohésion sociale rendent difficiles leur hiérarchisation. Tous sont à la fois englobés et englobant, spécifiques et globaux. Agir sur l'un provoque (en théorie) des répercussions sur les deux autres.

Les labels sont un exemple de l'appropriation depuis plus de 20 ans de ces différents concepts et de leur retranscription au domaine de la construction (figure 8). Ces derniers, de par leurs démarches volontaires, permettent de valoriser une démarche pro-active de prise en compte de l'environnement. En cela ils apparaissent, en complément des approches réglementaire et fiscales, comme des outils pouvant contribuer à l'atteinte du facteur 4 . 
Figure 8. Interconnexion des concepts et points d'entrées des labels

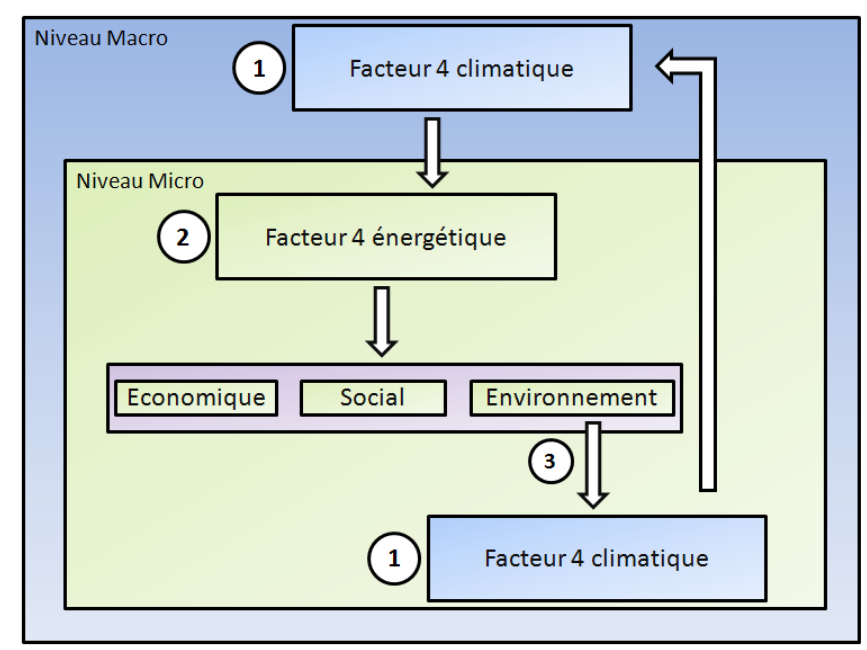

Légende Facteur 4 climatique Facteur 4 énergétique

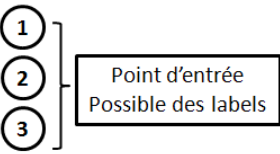

Source : Auteurs

\section{Les labels de la construction}

C'est dans un contexte mondial de consommation « frénétique » des ressources naturelles et de l'apparition de concepts tel que le «facteur 4 » (climatique ou énergétique) qu'il faut replacer l'apparition (figure 9) des labels de la construction.

Généralement issus d'initiatives portées par des acteurs locaux ou nationaux, et non d'une démarche de négociation internationale, les divers labels de la construction se sont insérés sur un marché mondial encore vierge. Portés par une volonté de standardisation et d'amélioration de la performance environnementale et énergétique, les labels ont cherché à standardiser des définitions de notions qui reposent sur des visions diverses, variant selon l'observateur, ses priorités ainsi que ses jugements de valeurs, mais également sur le contexte géographique, environnemental et culturel de chaque bâtiment. L'étude menée en partenariat avec l'Institut Français pour la Performance Énergétique des Bâtiment (Villot et al., 2009) a consisté à analyser 10 labels internationaux afin de comprendre leur fonctionnement, leurs objectifs, et d'analyser leur influence notamment pour l'atteinte des objectifs des facteurs 4 (énergétique et climatique). 
Figure 9. Échelle chronologique de création de quelques labels

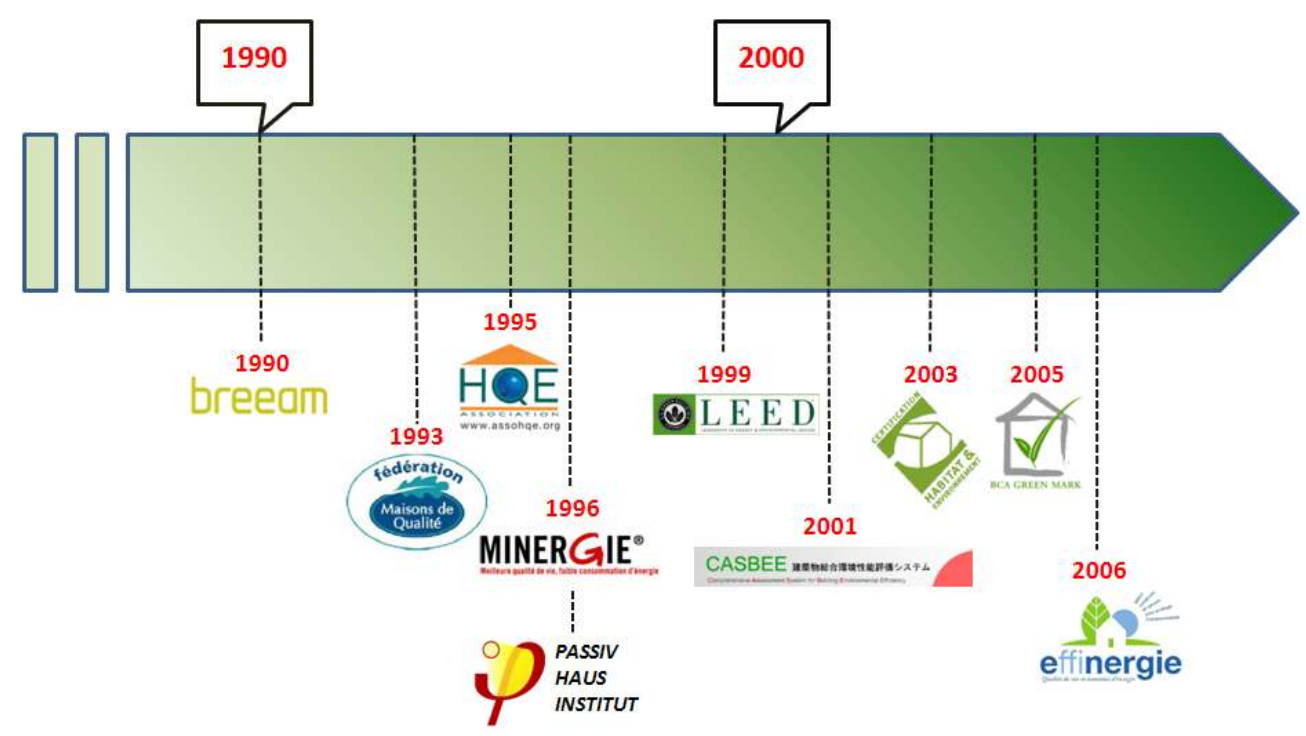

Source : Auteurs

\subsection{Le choix des référentiels et méthodes d'évaluation environnementale}

L'évaluation environnementale, dans le domaine de la construction, connait un plein essor depuis le début des années 1990. Au niveau mondial, l'étude a dénombré pas moins d'une soixantaine de méthodes d'évaluation ou d'analyse environnementale des bâtiments. L'analyse des documents se rapportant à ces méthodes nous a permis de les classer. "Globalement, il existe deux catégories principales de méthodes d'évaluation $»$ (Abdelghani-Idrissi et al., 2004) :

1- Les méthodes d'évaluation environnementale de type check-list ;

2- Les méthodes d'évaluation environnementale par analyse du cycle de vie (ACV).

Outre ces deux catégories, nous proposons d'étudier une troisième catégorie réunissant les méthodes d'évaluation qui proposent un système de notation et/ou de pondération reconnu à l'échelle d'un pays voire d'un groupe de pays : les méthodes d'évaluation par labellisation (Abdelghani-Idrissi et al., 2004). Le choix des méthodes s'est donc porté sur cette troisième catégorie en ciblant principalement les labels français et les labels étrangers dont la proximité géographique ou encore la portée internationale permettrait d'envisager une application en France (le 15 juin 2009, le BRE et le CSTB ont officialisé le projet d'alignement de la certification $\mathrm{HQE}$ et du label BREEAM). Suivant cette méthodologie, 10 labels ont été sélectionnés.

\section{Quatre labels français :}

1- Le label Haute Qualité Environnementale (HQE) (CERTIVEA, 2008) ;

2- Le label Habitat et Environnement (Qualitel, 2008);

3- Le label Maisons de Qualité (Envirobat, 2007);

Le label Bâtiment Basse Consommation (BBC) (Association Effinergie, 2008).

Deux labels à vocation internationale :

4- Le label Leadership in Energy and Environmental Design (LEED) (Canada Green 
Building Council, 2004);

5- Le label BRE Environmental Assessment Method (BREEAM) (BRE, 2008)

Quatre labels d'origine étrangère :

6- Le label Minergie (Association Minergie, 2008) ;

7- Le label Passivhaus (La maison passive France, 2007) ;

8- Le label Green Mark (Green Mark, 2008) ;

9- Le label CASBEE (JSBC, 2008).

31 Soulignons que cette étude ayant été menée de décembre 2008 à février 2009, seuls ont été pris en compte les labels publiés avant décembre 2008. C'est pourquoi la démarche «Bâtiment Durables Méditerranéens », par exemple, n'a pas pu être prise en compte dans cette étude. L'étude présentée dans cet article n'a en aucun cas l'objectif ou la prétention d'étudier en détail chaque label et encore moins de leur attribuer « une note». De fait, créer un modèle permettant une comparaison «objective» des labels semble difficilement réalisable. L'objectif de l'analyse des labels est donc de tenter d'identifier à partir d'indicateurs simplifiés, les grandes tendances, visions et objectifs (si différences il y a) de ces derniers.

\subsection{L'analyse des labels}

L'une des premières tâches inféodée à l'étude des labels consiste à s'attarder sur leurs structures, c'est-à-dire aux différentes strates hiérarchiques qui les composent. Il ressort ainsi que les systèmes d'agrégation et de pondération utilisés par les labels du bâtiment sont basés sur cinq niveaux d'analyse et de présentation des données (Boutaud, 2004) (figure 10) :

les données de base (rang 1) représentent l'ensemble des données statistiques et économiques généralement nécessaires aux spécialistes du domaine ;

les indicateurs unitaires qualitatifs ou quantitatifs (rang 2) sont calculés à partir des données de base et peuvent être utilisés par les décideurs ;

les sous-critères ou critères (rang 3) synthétisent les informations apportées par les indicateurs unitaires ;

les indices globaux ou thèmes (rang 4), dernière agrégation, permettent une meilleure diffusion des informations en ciblant les personnes extérieures au milieu d'étude ;

Les dimensions (rang 5), agrégation optionnelle permettant une représentation simplifiée du champ d'investigation des labels. 


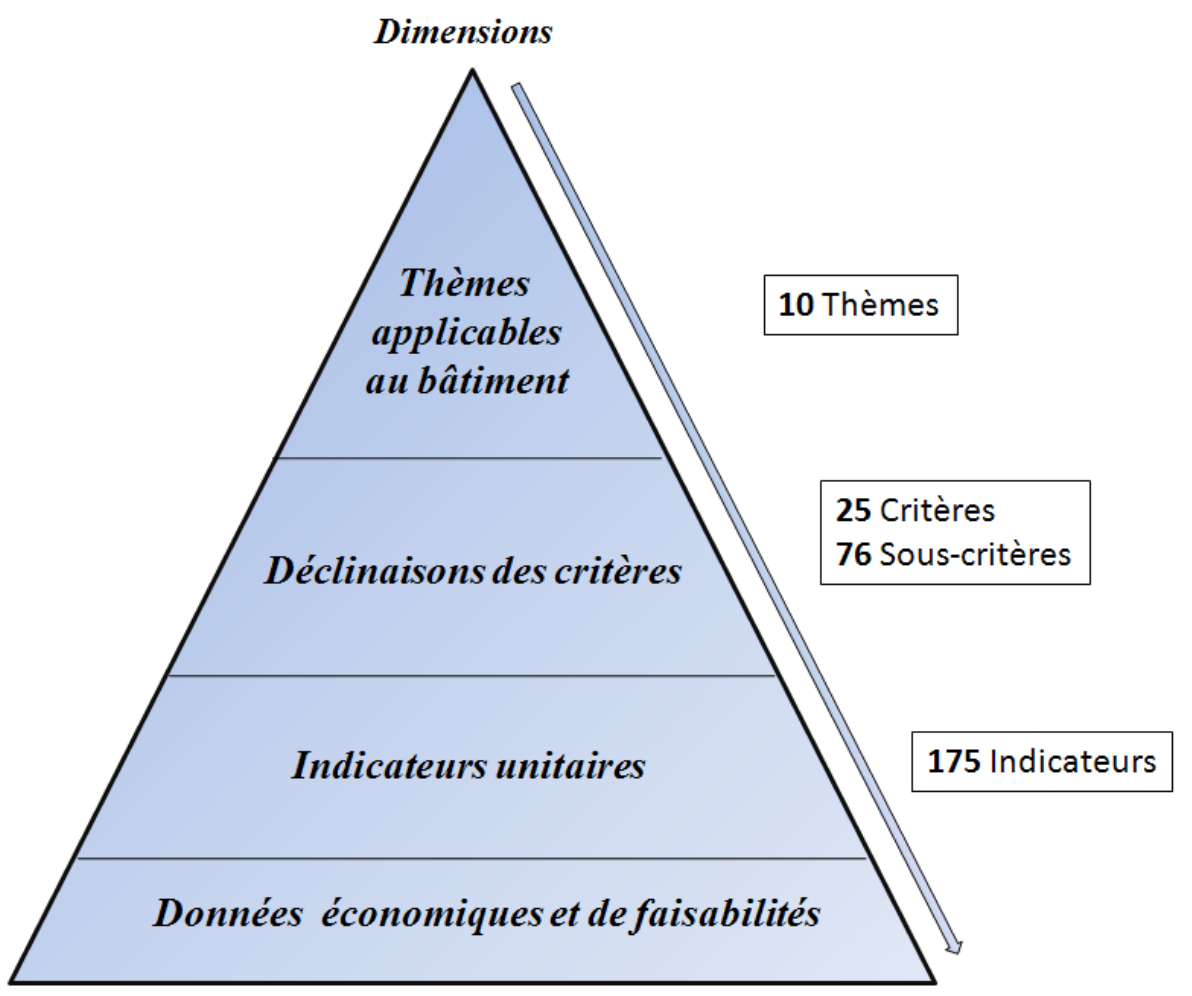

Source : Auteurs d'après Boutaud, 2004

Pour cette étude, la catégorie ciblée est le rang 3 de cette classification (figure 11), c'est-àdire les sous-critères utilisés par les labels. De fait, les données de rang inférieur, de par leur multiplicité et leur aspect souvent technique, sont trop précises pour faire ressortir clairement la vision portée par les labels. Inversement, les niveaux supérieurs par un « corsetage » important du fait des pondérations et agrégations successives sont trop vagues pour différencier les messages portés par les labels. Le phénomène de corsetage permet « selon le point de vue de l'observateur et ses propres priorités de filtrer les informations, conduisant à ce que différents publics concernés tirent des conclusions différentes d'une même base d'information" (NF ISO 15392, 2008). Ce phénomène très important pour la comparaison des labels est donc à prendre en considération, sachant qu'à des niveaux inférieurs (rang 3), il différenciera les labels alors qu'une utilisation abusive (rang 4 et 5) aura tendance à les homogénéiser. 
Figure 11. Hiérarchisation des niveaux dans les labels de la construction

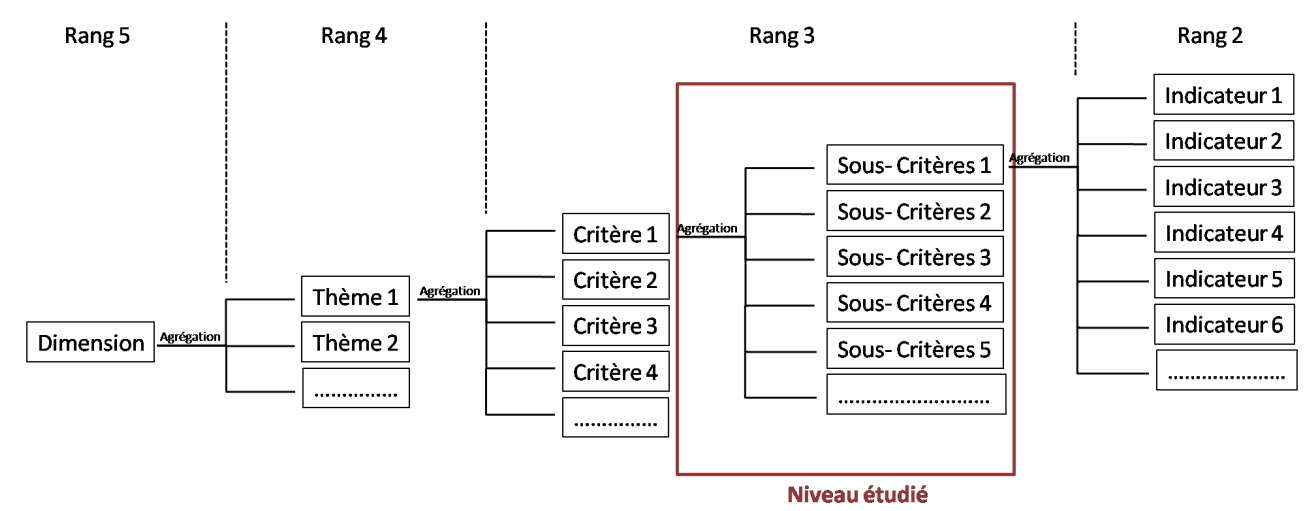

Source : Auteurs, d'après Boutaud, 2004

Par la suite, les 76 sous-critères recensés ont été classés. Pour cela, la méthodologie d'agrégation (réalisée d'après ISO/TS 21929-1) a conduit à la création de cinq dimensions (Villot et al., 2009) :

D1: Changement climatique : participation du bâtiment au changement climatique, en termes d'émissions de GES. Comptabilité concernant l'intégralité du bâtiment: déplacements induits, GES issus des matériaux ou de l'utilisation du bâtiment.

$\rightarrow$ Exemples de sous-critères appartenant à la dimension D1 : réduction des émissions de GES, mise en place de transports doux, ...

D2 : Ressources naturelles : effort de maîtrise des consommations de ressources naturelles pour la construction et l'utilisation du bâtiment, volonté de privilégier les ressources renouvelables au niveau planétaire: matériaux de construction renouvelables, ou de filières éco gérées, ressources énergétique ou aquatique préservées (renouvelable ou bilan équilibré).

$\rightarrow$ Exemples de sous-critères appartenant à la dimension D2: qualité environnementale des matériaux et des produits, réduction des consommations d'énergie, ...

D3 : Habitat et l'utilisateur : prise en compte des habitants dans tous les aspects de leur humanité (confort, qualité sanitaire, acoustique, praticité des lieux, management participatif et prise en compte des nécessités spécifiques possibles).

$\rightarrow$ Exemples de sous-critères appartenant à la dimension D3 : gouvernance et sensibilisation des habitants, convertibilité des logements et des espaces en commun, ...

D4: Société: intelligence des lieux compte tenu de leur destination, intégration urbanistique, beauté, inspiration, etc.

$\rightarrow$ Exemples de sous-critères appartenant à la dimension D4: durabilité face au temps, qualité fonctionnelle du site, ...

40 D5 : Économie : prix et accessibilité du logement et de son utilisation.

$\rightarrow$ Exemples de sous-critères appartenant à la dimension D5 : surcoût du bâtiment, répercutions des surcoûts sur les loyers, ...

L'objectif de ce classement n'est pas de créer un nouveau label, fruit de la fusion des 10 labels sélectionnés précédemment mais de permettre une "cartographie» de ces derniers. De fait l'agrégation des sous-critères dans les 5 dimensions réintroduit le phénomène de corsetage évoqué ci-dessus ainsi qu'une part de subjectivité. En 
contrepartie, elle permet une représentation intuitive du champ d'investigation des labels.

\subsection{Les sous-critères, cœur des labels}

Chaque label a une vision (et ou un objectif), portée majoritairement par le choix de ses critères et sous-critères mais aussi par leur importance relative. Durant l'étude, plusieurs niveaux d'utilisation des sous-critères ont été différenciés (tableau 1).

Tableau 1. Niveaux d'utilisation des sous-critères et définitions

\begin{tabular}{|l|l|}
\hline $\begin{array}{l}\text { Niveaux } \\
\text { d'utilisation }\end{array}$ & Définitions \\
\hline Non Traité & Sous-critère non traité par le label \\
\hline Recommandé & Sous-critère préconisé par le label (Ni obligatoire, ni pondéré) \\
\hline Implicite & $\begin{array}{l}\text { Sous-critère du label non cité mais s'exprimant à travers un autre Sous- } \\
\text { critère }\end{array}$ \\
\hline Pondéré & Sous-critère du label non obligatoire mais ayant un poids dans la notation \\
\hline Fondamental & Sous-critère du label de traitement obligatoire \\
\hline
\end{tabular}

Source : Auteurs

L'obtention des labels étant conditionnée par la validation des sous-critères pondérés et fondamentaux, une attention particulière leur a été accordée. Dans ce but, une quantification de ces deux types de sous-critères a été réalisée (figure 12). Tous les labels ne considèrent pas de la même façon un même sous-critère (pondéré ou fondamental). Il est important de notifier que le type de sous-critère n'a aucune influence sur la performance exigée par ce sous-critère. À titre d'exemple, le sous-critère "maîtrise des consommations de chauffage» sera considéré d'après notre classement comme fondamental pour BBC mais comme pondéré dans les labels CASBEE ou BREEAM qui n'imposent pas la prise en compte obligatoire de ce critère. Sur ce point, depuis peu, le label HQE adopte une position originale : son sous-critère " maitrise des consommations de chauffage ", qui se trouve dans la cible 4 du label, fait appel à d'autres démarches de labellisation : labels THPE ou BBC (Effinergie). Ainsi, cette cible doit « obligatoirement être atteinte à un niveau Performant ou Très Performant, pour les bâtiments ou parties de bâtiments ayant l'obligation de respecter la Règlementation Thermique en vigueur. Dans les autres cas, on pourra se contenter de l'atteinte du niveau Base. » Les niveaux peuvent aller de la très haute performance énergétique (THPE) au bâtiment à énergie positive (CERTIVEA, 2008).

De par cette classification des sous-critères, trois groupes de labels sont alors identifiables (figure 12) :

Les labels « contraignants »: $100 \%$ de sous-critères obligatoires (fondamental) ; les labels « à choix restrictifs »: Partage entre les sous-critères pondérés et obligatoires ; les labels « non-directifs » : $100 \%$ de sous-critères pondérés. 
Figure 12. Proportion de sous-critères fondamentaux et pondérés au sein des labels

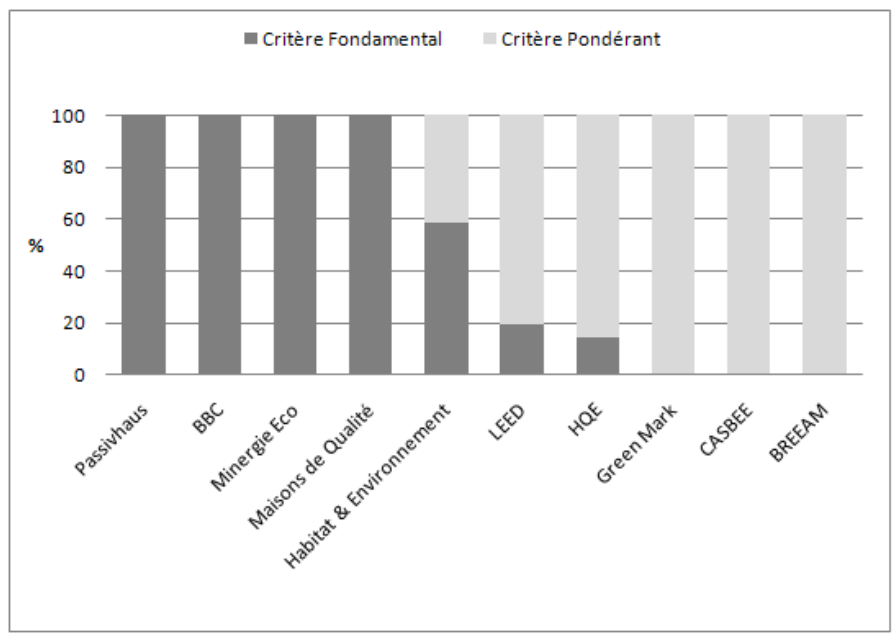

Source : Auteurs

Dans un second temps, et en se basant sur les 5 dimensions évoquées précédemment (§3.2), nous avons « cartographié » le champ d’investigation de ces labels. Pour cela nous avons relevé les sous-critères de l'ensemble des labels en écartant les données redondantes. Par la suite chaque sous-critère a été agrégé dans une des 5 dimensions (un même sous-critère pouvant appartenir à plusieurs dimensions). Pour finir, nous avons quantifié la proportion de sous-critères dans chaque label par rapport à l'ensemble des sous-critères existants (sous-critères de l'ensemble des labels). Le résultat de cette approche est représenté par la figure 13.

Figure 13. Cartographie des labels selon 5 dimensions du développement durable

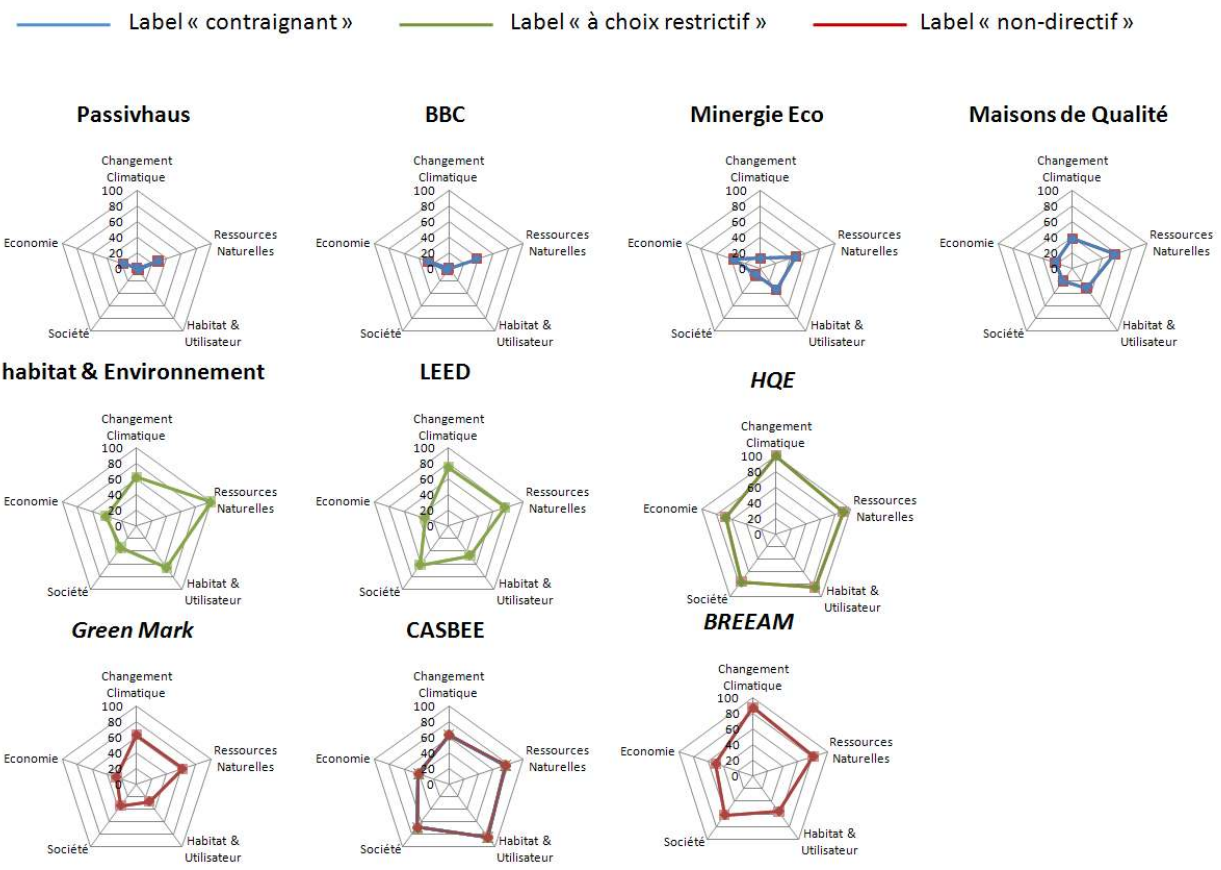

Source : Auteurs 
maraison des figures 12 et 13 montre une corrélation négative entre le nombre de dimensions et donc le nombre de sous-critères abordés par un label et la proportion (en pourcent) de sous-critères fondamentaux obligatoires. De fait, les labels " non-directifs » (CASBEE, BREEAM) ne possédant aucun sous-critère fondamental, comptent une grande variété de sous-critères et évoluent généralement dans les cinq dimensions. En revanche, les labels «contraignants » (BBC, Passivhaus) basés exclusivement sur des sous-critères obligatoires n'abordent que quelques sous-critères et évoluent dans une ou deux dimensions.

Encadré 1. Une hiérarchisation à éviter

La figure 13 représente tous les sous-critères sans les distinguer qu'ils soient obligatoires ou pondérés et sans niveaux de performance. Ainsi, il faut éviter la hiérarchisation des labels à partir de ces figures. En effet, cette hiérarchisation à partir des données présentées serait fausse et ce pour deux raisons. D'une part, il n'y a dans la figure 13 aucune distinction entre critère pondéré et obligatoire et, d'autre part, le niveau demandé pour répondre à un critère varie énormément selon les labels et n'est pas pris en compte à ce stade de l'étude.

Ce sont donc les types de sous-critères et les libertés (label « non-directif ») et restrictions (label «contraignant») qui orientent la vision des labels et les segmentent en deux catégories : la première essayant d'englober la plupart des dimensions, l'autre focalisée sur les aspects énergétiques (ressources naturelles) et économiques. Cependant, si les labels de la seconde catégorie communiquent sur l'efficacité énergétique en " prônant » un objectif optimal, les labels «non-directifs » sont principalement basés sur les aspects environnementaux de la construction et de part leurs dimensions communiquent plus sur un moyen d'atteindre un objectif que sur l'objectif lui même. Ce phénomène est observable directement par l'analyse du nom des labels (tableau 2). Pour plus de facilité, les labels communiquant sur l'environnement seront appelés labels environnementaux alors que ceux communiquant sur l'énergie seront nommés labels énergétiques.

Tableau 2. Analyse des noms des labels

\begin{tabular}{|l|l|}
\hline PASSIVHAUS : Maison passive (énergétiquement) & $\begin{array}{l}\text { Référence aux aspects } \\
\text { énergétiques }\end{array}$ \\
\hline BBC : Bâtiment Basse consommation (en énergie) & $\begin{array}{l}\text { Référence aux aspects } \\
\text { énergétiques et } \\
\text { environnementaux }\end{array}$ \\
\hline Maison de qualité (pas de référence clair aux aspects énergétiques) \\
\cline { 1 - 1 } LEED: Leadership in Energy and Environmental Design & $\begin{array}{l}\text { Référence aux aspects } \\
\text { environnementaux }\end{array}$ \\
\cline { 1 - 1 } $\begin{array}{l}\text { CASBEE: Comprehensive Assessment System for Building } \\
\text { Environmental }\end{array}$ & \\
\hline BREEAM: BRE Environmental Assessment Method & \\
\hline HQE : Haute Qualité Environnementale & \\
\hline Green Mark &
\end{tabular}

Source : Auteurs

Il apparait alors à ce niveau que les labels envisagent la construction selon deux approches ou rationalités : l'une substantive, l'autre procédurale. 


\subsection{Des rationalités et des labels}

49 Le concept de rationalité, introduit par Herbert A. Simon s'applique «au choix des alternatives qui seront préférées en fonction d'un système de valeurs permettant d'évaluer les conséquences du comportement choisi». Deux grandes rationalités du comportement ont été étudiées par Simon : la rationalité substantive (ou substantielle) et la rationalité procédurale.

50 « Le comportement est substantivement rationnel quand il est en mesure d'atteindre les buts donnés à l'intérieur des limites imposées par les conditions et les contraintes données. Notons que, par définition, la rationalité du comportement ne dépend de l'acteur que d'un seul point de vue - celui des buts. Une fois ces buts fixés, le comportement rationnel est entièrement déterminé par les caractéristiques de l'environnement dans lequel il a lieu. » (Simon, 1992).

51 La rationalité procédurale quant à elle ne s'attache pas tant à l'atteinte d'un objectif mais plutôt à la démarche mise en place pour l'atteindre. Il s'agit «d'attirer l'attention sur certains aspects de la situation au détriment d'autres points susceptibles d'orienter le choix dans une direction différente » (Simon, 1947). De plus, «la recherche de solutions ne se poursuit pas jusqu'à une solution optimale; elle s'arrête lorsque l'agent trouve une solution qui lui semble pouvoir satisfaire ses besoins, c'est-à-dire une solution qui semble correspondre à son niveau d'aspiration" (Parthenay, 2005). Il ressort de ceci que la rationalité procédurale est plutôt porté sur les moyens d'atteindre un but et non sur le but lui-même.

52 Appliqué aux labels, nous remarquons que, les labels énergétiques communiquent sur un objectif précis souvent considéré comme optimal (rationalité substantive) alors que les labels environnementaux, dans la lignée des outils de management environnemental, définissent plutôt une démarche, un ensemble d'actions permettant d'atteindre des objectifs fixés par l'organisation elle-même (rationalité procédurale). De part ce constat, une comparaison des labels sur l'ensemble de leurs thématiques semble difficile. Ainsi, compte-tenu de notre volonté d'envisager le rôle des labels dans l'optique du facteur 4 , nous situerons plutôt notre analyse dans une perspective de rationalité substantive et étudierons la prise en compte de l'objectif du facteur 4 à travers le thème commun à tout les labels : l'énergie.

\section{Les labels de la construction et leur contribution aux facteurs 4}

\subsection{Les labels énergétiques}

Les labels de la construction, et plus particulièrement les labels énergétiques, montrent un intérêt généralisé pour la maîtrise de l'énergie dans le bâtiment. Les objectifs visés varient cependant selon les labels, contribuant plus ou moins à l'atteinte du facteur 4 énergétique et climatique. Ce constat peut être établi en étudiant les labels dits " énergétiques». Parmi les 10 labels étudiés, trois entrent dans cette catégorie: Passivhaus, BBC et Minergie. Selon la loi dite «Grenelle 1 », pour respecter le facteur 4 énergétique et climatique, chaque bâtiment devra consommer moins de $60 \mathrm{kWh}_{\mathrm{ep}} / \mathrm{m}^{2} / \mathrm{an}$ (Chauffage+ECS) en 2050, et émettre au maximum $9 \mathrm{kgCO}_{2} / \mathrm{m}^{2} /$ an (hypothèse de division 
par 4 par rapport aux émissions actuelles) (Grenelle Groupe 1, 2008b). Comparer les labels par rapport à cet objectif est particulièrement difficile étant donné que les postes d'émissions et de consommations ainsi que les méthodes de calcul des besoins énergétiques ne sont pas les mêmes (tableau 3). Cependant, en posant l'hypothèse qu'un label ayant un objectif inférieur à $60 \mathrm{kWhep} / \mathrm{m} 2 / \mathrm{an}$, pour un nombre de poste de consommation comprenant au moins tous les usages thermiques de l'énergie (chauffage, ECS, ventilation et climatisation,) permet de répondre au facteur 4, alors les labels Minergie et Effinergie en font partie.

Tableau 3. Consommations et usages concernés

\begin{tabular}{|c|c|c|c|c|}
\hline & Minergie & Effinergie $\mathrm{BBC}$ & Passivhaus & $\begin{array}{l}\text { Facteur } \quad 4 \\
(2050)\end{array}$ \\
\hline $\begin{array}{ll}\text { Seuil } & \text { de } \\
\text { consommations } & \\
\text { bâtiment neuf } & \end{array}$ & $\begin{array}{l}38 \mathrm{kWh}_{\mathrm{ep}} / \mathrm{m}^{2} \\
/ \mathrm{an}\end{array}$ & $50 \mathrm{kWh}_{\mathrm{ep}} / \mathrm{m}^{2} / \mathrm{an}$ & $120 \mathrm{kWh}_{\mathrm{ep}} / \mathrm{m}^{2} / \mathrm{an}$ & $\begin{array}{l}60 \mathrm{kWh}_{\mathrm{ep}} / \\
\mathrm{m}^{2} / \mathrm{an}\end{array}$ \\
\hline $\begin{array}{l}\text { Seuil d'émissions } \\
\text { équivalent* }\end{array}$ & $\begin{array}{l}6 \mathrm{kgCO}_{2} / \mathrm{m}^{2} / \\
\text { an }\end{array}$ & $7 \mathrm{kgCO}_{2} / \mathrm{m}^{2} / \mathrm{an}$ & $17,5 \mathrm{kgCO}_{2} / \mathrm{m}^{2} / \mathrm{an}$ & $\begin{array}{l}9 \mathrm{kgCO}_{2} / \mathrm{m} \\
2 / \mathrm{an}\end{array}$ \\
\hline $\begin{array}{l}\text { Usage concernée pour } \\
\text { les seuils de } \\
\text { consommations }\end{array}$ & $\begin{array}{l}\text { Chauffage } \\
+ \text { ECS } \\
+ \text { Ventilation } \\
+ \\
\text { Climatisation }\end{array}$ & $\begin{array}{l}\text { Chauffage } \\
+ \text { ECS } \\
+ \text { Ventilation } \\
+ \\
\text { Refroidissement } \\
+ \text { Éclairages } \\
+ \text { Auxiliaires } \\
\text { - Photovoltaïque }\end{array}$ & $\begin{array}{l}\text { Toutes consommations } \\
\text { d'énergie, énergie } \\
\text { spécifique incluse }\end{array}$ & $\begin{array}{l}\text { Chauffage } \\
+ \text { ECS }\end{array}$ \\
\hline
\end{tabular}

54 * hypothèse de $14 \mathrm{~g}$ de $\mathrm{CO}_{2}$ par kWhep

Source : auteurs à partir du site de www.alterre-bourgogne.fr

À première vue, le label Passivhaus semble exclu. Cependant, ce label prend en compte tous les postes de consommation et est donc plus exhaustif que d'autres. De plus, il fixe une limite en besoin de chauffage à $15 \mathrm{kWhEU} / \mathrm{m}^{2} / \mathrm{an}$ (EU = Énergie Utile). À titre de comparaison et en utilisant les données du logiciel 3CL et les chaudières les plus efficaces, une consommation d'énergie primaire de $50 \mathrm{kWhep} / \mathrm{m}^{2} /$ an correspond à un besoin de chauffage d'environ $35 \mathrm{kWhEU} / \mathrm{m}^{2} / \mathrm{an}$. Ainsi, le label Passivhaus semble plus exigeant. Ces résultats sont à modérer du fait des différences de méthodes de calculs entre labels et entre pays. En effet, chaque pays et label ont des conventions pouvant grandement modifier l'expression de la performance du bâtiment et donc nuire à la compréhension de l'objectif du facteur 4.

Tous les labels, énergétiques comme environnementaux, expriment les consommations énergétiques des bâtiments en kWh. Cependant, selon les cas, ces données peuvent faire référence à 3 notions distinctes : l'énergie primaire, l'énergie finale et l'énergie utile.

1- L'énergie primaire « est la première forme de l'énergie directement disponible dans la 
nature: bois, charbon, gaz naturel, pétrole, vent, rayonnement solaire, énergie hydraulique, géothermique... L'énergie primaire n'est pas toujours directement utilisable et fait donc souvent l'objet de transformations : par exemple, raffinage du pétrole pour avoir de l'essence ou du gazole; combustion du charbon pour produire de l'électricité dans une centrale thermique ";

2- L'énergie finale "est l'énergie livrée aux consommateurs pour être convertie en énergie utile : par exemple : électricité, essence, gaz, gazole, fioul domestique, etc. » ;

3- L'énergie utile « est l'énergie dont dispose le consommateur, après transformation par ses équipements (chaudière, convecteurs électriques, ampoule électrique). La différence entre l'énergie finale et l'énergie utile tient essentiellement au rendement des appareils utilisés pour transformer cette énergie finale ».

Ces trois énergies sont toutes reliées entre elle par les équations suivantes (équation 1) :

Équation 1. Équations simplifiées pour le calcul de la consommation de chauffage (TRIBU Énergie, 2006).

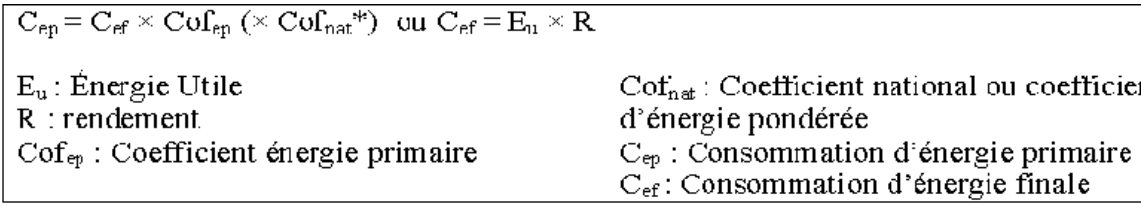

Encadré 2. Cof $_{\text {ep }}$ et Cof $_{\text {nat }}$

Le $\mathrm{Cof}_{\mathrm{ep}}$ est un coefficient utilisé pour le passage des consommations d'énergie finale en énergie primaire. Ce coefficient est calculé en prenant compte toute les transformations de l'énergie (cf. énergie primaire). Le Cof ${ }_{\text {nat }}$ ou coefficient d'énergie pondérée (SIA, 2009) est utilisé quant à lui pour promouvoir une énergie. Ainsi dans certains labels, le Cof est $_{\text {est }}$

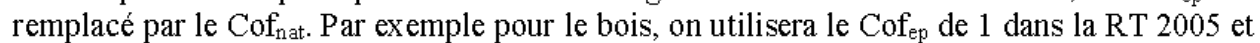



Par ces équations, on peut observer que de nombreuses variables interviennent pour le calcul de la consommation d'énergie primaire. Certains coefficients sont issus de données physiques ( $\mathrm{R}$ ) alors que d'autres sont issus des conventions politiques et des mix énergétiques propres à chaque pays $\left(\mathrm{Cof}_{\mathrm{ep}}, \mathrm{C}_{\mathrm{nat}}\right)$. Retranscrites au niveau des labels (tableau 4), ces conventions peuvent grandement modifier les affichages des consommations d'énergie des bâtiments. 
Tableau 4. Conversion Énergie Primaire-Énergie Finale

\begin{tabular}{|l|l|l|l|l|}
\hline & RT 2005 & Minergie & Passivhaus & Effinergie BBC \\
\hline Électricité & 2,58 & 2 & 2,7 & 2,58 \\
\hline Énergie fossile & 1 & 1 & 1,1 & 1 \\
\hline Bois & 1 & 0,5 & 0,2 & 0,6 \\
\hline Photovoltaïque & $/$ & 2 & 0,7 & 1 \\
\hline
\end{tabular}

Source : auteurs à partir du site www.alterre-bourgogne.fr

$$
\text { Àt titse }
$$
par Passivhaus en Allemagne. Au contraire, avec une source d'énergie électrique, le même bâtiment apparaitra comme plus consommateur en Allemagne qu'en France! Pour simplifier, le même bâtiment dans les mêmes conditions climatiques n'aura pas les mêmes affichages d'impact sur les consommations d'énergie et les émissions de GES selon les labels et les pays. L'énergie bois est l'exemple le plus frappant de la mise en place d'un coefficient national, coefficient attribué plus ou moins «arbitrairement " par les pays. Cela s'explique par les faibles émissions de $\mathrm{CO}_{2}$ par $\mathrm{kWh}$ de l'énergie bois comparées notamment au fioul, au gaz, ou encore à l'énergie électrique $\left(2,5\right.$ fois plus de $\mathrm{CO}_{2}$ par $\mathrm{kWh}$ ep que le bois (ADEME, 2007). Cette démarche s'insère dans la politique du facteur 4 énergétique et climatique; cependant elle se révèle à double tranchant. De fait, elle permet la promotion de l'énergie bois, et donc la réduction de l'affichage des émissions de $\mathrm{CO}_{2}$, en réduisant de 50 à $80 \%$ les émissions et les consommations théoriques. Cependant, les émissions réelles ne sont aucunement influencées par les pondérations humaines et donc l'application de coefficients nationaux quelque soit le type d'énergie n'est pas une solution à long terme pour l'atteinte du facteur 4.

60 Les labels contournent donc les réalités physiques en intégrant de façon détournée des systèmes de pondérations permettant de sublimer les caractéristiques des bâtiments. L'influence des labels et leur contribution aux facteurs 4 peuvent donc varier selon leurs objectifs, le pays d'origine et les conventions de calcul (pondération).

\subsection{Les labels environnementaux}

61 Les labels environnementaux, grâce à des démarches multicritère sont désignés comme les seuls à viser à la prise en compte de l'ensemble des objectifs de la construction souvent définie comme « durable » (Ding, 2008). Cependant, ils n'exigent pas pour autant l'atteinte des objectifs du facteur 4 énergétique et climatique. Bien sûr, la prise en compte de plusieurs des 5 dimensions évoquées précédemment permet de se rapprocher d'une démarche "complète "; mais l'exhaustivité de cette approche a pour contrepartie de laisser un maximum de liberté au maître d'ouvrage. En effet, la majorité des labels environnementaux utilise des critères pondérés. La pondération utilisée sur un critère ou un groupe de critères permet au demandeur du label de choisir quels sont pour lui les 
objectifs primordiaux pour la réussite de son projet de construction (rationalité procédurale). Cette liberté pouvant dans certains cas estomper l'importance de la performance notamment énergétique et environnementale du bâtiment. Le label BREEAM en est un exemple.

Tableau 5. Exemple de notation d'un projet sous BREEAM

\begin{tabular}{|c|c|c|c|c|c|}
\hline BREEAM Section & 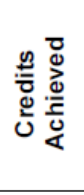 &  & 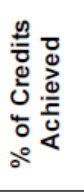 & 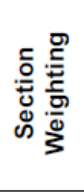 & 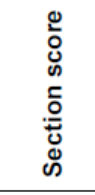 \\
\hline Management & 7 & 10 & $70 \%$ & 0.12 & $8.40 \%$ \\
\hline Health \& Wellbeing & 11 & 14 & $79 \%$ & 0.15 & $11.79 \%$ \\
\hline Energy & 10 & 21 & $48 \%$ & 0.19 & $9.05 \%$ \\
\hline Transport & 5 & 10 & $50 \%$ & 0.08 & $4.00 \%$ \\
\hline Water & 4 & 6 & $67 \%$ & 0.06 & $4.00 \%$ \\
\hline Materials & 6 & 12 & $50 \%$ & 0.125 & $6.25 \%$ \\
\hline Waste & 3 & 7 & $43 \%$ & 0.075 & $3.21 \%$ \\
\hline Land Use \& Ecology & 4 & 10 & $40 \%$ & 0.10 & $4.00 \%$ \\
\hline Pollution & 5 & 12 & $42 \%$ & 0.10 & $4.17 \%$ \\
\hline & & \multicolumn{2}{|c|}{ Total Score } & \multicolumn{2}{|c|}{$54.87 \%$} \\
\hline & \multicolumn{3}{|c|}{ Innovation credits achieved } & \multicolumn{2}{|c|}{1} \\
\hline & \multicolumn{3}{|c|}{ FINAL BREEAM Score } & \multicolumn{2}{|c|}{$55.87 \%$} \\
\hline & \multicolumn{3}{|c|}{ BREEAM Rating } & \multicolumn{2}{|c|}{ VERY GOOD } \\
\hline
\end{tabular}

Source : BRE, 2008

Sur le tableau 5 est représenté le système de labellisation de BREEAM. La note finale (Very GOOD Score final $\geq 55 \%$ ) est obtenue par la somme des crédits pondérés du poids de chaque section. Un même score final peut donc être obtenu par plusieurs configurations différentes. Ce système risque donc de diluer les questions énergétiques (consommation d'énergie) et climatiques $\left(\mathrm{CO}_{2}\right)$ et donc les objectifs des facteurs 4. Par exemple, la section énergie base ses critères sur la diminution de l'impact environnemental - dont la valeur dérive des émissions de $\mathrm{CO}_{2}$ en $\mathrm{kgCO}_{2} / \mathrm{m}^{2}$ - et montre donc le lien implicite entre consommation d'énergie et émissions de $\mathrm{CO}_{2}$. Cependant, aucun niveau n'est imposé pour les émissions de $\mathrm{CO}_{2}$ permettant au demandeur du label de choisir le niveau qui lui convient (à condition de respecter les normes réglementaires si elles existent). Ainsi, l'obtention du niveau maximum chez BREEAM ne garantit pas forcément l'équivalent "platinium» par le label LEED. Les labels "environnementaux» peuvent donc être considérés comme un moyen de sensibilisation aux aspects énergétiques et climatiques mais leur pondération et l'absence d'objectifs chiffrés ne garantissent en aucun cas l'atteinte des objectifs des facteurs 4. 


\section{Les oubliés de la labellisation dans la perspective du facteur 4}

Les systèmes de labellisation sont des systèmes plus ou moins complexes faisant intervenir une thématique ou un ensemble de critères diversifiés. Visant des objectifs précis ou plus larges, ils oublient ou ignorent des aspects de la construction dont le rôle est prépondérant pour l'atteinte des objectifs des facteurs 4.

\subsection{Les étapes de la labellisation}

Un bâtiment peut être labellisé à différentes étapes de son cycle de vie. De la planification $\mathrm{du}$ projet à son fonctionnement en passant par les phases de conception et de construction, les moments de la labellisation jouent un rôle très important sur les résultats et objectifs attendus. Le regroupement des labels selon ces phases montrent le délaissement des phases de planification et de fonctionnement pour un certain nombre de labels (tableau 6).

Tableau 6. Étapes de l'évaluation par les labels

\begin{tabular}{|c|c|c|c|c|}
\hline Label & Planification & Conception & Construction & Fonctionnement \\
\hline PASSIVHAUS & & . & & \\
\hline $\mathrm{HQE}$ & - & - & - & \\
\hline LEED & & & - & . \\
\hline MAISON DE QUALITE & & & - & \\
\hline$H \& B$ & - & - & . & \\
\hline $\mathrm{BBC}$ & $\cdot$ & - & . & \\
\hline MINERGIE ECO & & - & - & . \\
\hline CASBEE & & - & $\cdot$ & $\cdot$ \\
\hline BREEAM & & - & & $\cdot$ \\
\hline GREEN MARK & - & . & & . \\
\hline
\end{tabular}

Source : Auteurs

La phase de fonctionnement correspond à «l'après construction ", c'est-à-dire l'étape où la théorie est confrontée à la pratique. Deux arguments peuvent être avancés pour expliquer l'absence d'évaluation à cette étape. La première raison est la durée d'étude. En effet, une année voire deux de mesures sont nécessaires à la vérification des données théoriques calculées lors des précédentes phases. La seconde explication est souvent la sous-estimation de l'impact des utilisateurs sur le bâti. Pour de nombreux labels, 
l'amélioration de l'enveloppe du bâti est la seule à pouvoir efficacement diminuer la consommation de l'habitat. Or, cette amélioration influe quasi uniquement sur les besoins intrinsèques au logement selon des scénarios d'utilisation génériques. Le scénario de chauffage est emblématique de la discordance entre théorie et pratique. En effet, les scénarios standards simulent des températures de chauffe de $19^{\circ} \mathrm{C}$ or en pratique les températures mesurées in-situ montrent un écart de $+2^{\circ} \mathrm{C}$. Cette différence est d'autant plus importante que l'augmentation de $1^{\circ} \mathrm{C}$ de la température de chauffe d'un bâtiment moyennement isolé, augmente de 5 à $8 \%$ la consommation d'énergie de ce dernier (INSEE, 2009). De ce fait, les avantages apportés par la mise en place d'une technologie (amélioration de l'enveloppe) peuvent être selon les cas partiellement ou totalement compensés par une modification du comportement de l'utilisateur. Cette idée est formalisée par la notion d'effet rebond ou "l'augmentation de consommation est liée à la réduction des limites à l'utilisation d'une technologie, ces limites pouvant être monétaires, temporelles, sociales, physiques, liées à l'effort, au danger, à l'organisation... " (Schneider, 2003). Ainsi, l'absence d'évaluation en fonctionnement semble regrettable au vu, d'une part, des possibilités d'accéder à des données de terrain sur le retour d'expérience des méthodes et techniques utilisées par les bâtiments labellisés (fiabilité sur le long terme, performances réelles par rapport aux performances attendues, etc.) et, d'autre part, d'un suivi de l'efficacité réelle, en termes énergétiques, des outils de labellisation " (Villot et al., 2009). Les possibles variations dans les objectifs ont de plus des conséquences sur les aspects financiers, aspects qui ne sont généralement pas abordés par les labels.

\subsection{Les aspects financiers de la labellisation}

66 Les méthodes de labellisation ont un coût, qui peut provenir de la certification ou du surinvestissement que provoque l'amélioration des performances du bâti. Malgré cela, les aspects financiers ne sont que très rarement évalués par les labels, alors que les aspects financiers sont fondamentaux durant les projets. «De fait, un projet peut être rentable d'un point de vue environnemental mais inabordable du point de vue économique» (Ding, 2008). Ce manque est surtout préjudiciable aux labels environnementaux qui ne suscitent pas le même intérêt ni le même « retour sur investissement » sur le long terme que les labels énergétiques. En effet, aux USA, sur le marché de la location, les bâtiments certifiés par un label énergétique peuvent voir leur valeur par $\mathrm{m}^{2}$ augmenter de 3 à $6 \%$, alors qu'aucun effet n'est constaté, sur la valeur locative, pour les labels environnementaux. "Cela suggère que les investisseurs sont prêts à payer plus pour de l'efficacité énergétique mais pas pour une construction "durable" au sens large" (Eichholtz et al., 2009). Ainsi, le facteur 4 énergétique semble plus convoité et accessible par des retours sur investissement que le facteur 4 climatique ou encore le concept de construction dite « durable ».

\subsection{Une reproductibilité limitée}

67 Les labels, notamment énergétiques ont une "durée de vie» limitée. Cela est particulièrement vrai pour le label BBC dont le niveau de performance va être généralisé en France par la future réglementation thermique (RT 2012). De ce fait, les labels sont une vitrine, une " rampe de lancement " pour l'amélioration du bâti. Cependant, le coût de la labellisation ou la complexité des critères limitent leur utilisation. En tant que nouvelle valeur dont l'utilisation est signe de progrès, la majorité des labellisations porte sur des constructions neuves. De fait, et malgré l'ouverture de nombreux labels au domaine de la 
rénovation du bâti existant, peu de projets ont été lancés sur ce secteur spécifique. Or le parc de logements anciens est majoritaire en France tant en nombre de logements (64,7\% des logements datent d'avant 1975) (Observatoire de l'énergie, 2009), qu'en consommation d'énergie ( $70,1 \%$ des consommations de chauffage). De ce fait, pour de nombreux experts, l'atteinte des objectifs du facteur 4 énergétique et climatique dans le secteur du bâtiment passe par l'obligation de rénovation massive du parc ancien (400 000 rénovations par an) (Sidler, 2007). L'atteinte «de ces objectifs passe par l'obligation de travaux. Choisir l'incitation plutôt que la réglementation conduira à une situation grave et irréversible: les bâtiments insuffisamment isolés (ex: $100 \mathrm{kWh} / \mathrm{m}^{2} / \mathrm{an}$ ) ne pourront plus faire l'objet de travaux complémentaires, impossibles à rentabiliser. Le potentiel actuel d'économie sera donc détruit de façon irréversible » (Sidler, 2003). Les labels par leurs aspects volontaires ont un impact limité à quelques bâtiments et n'ont donc pas les moyens d'atteindre l'ensemble des bâtiments ce qui éloigne l'ensemble du parc résidentiel des objectifs du facteur 4 énergétique et climatique.

\section{Conclusion}

Au cours des 20 dernières années, le concept de facteur 4 a connu des évolutions. Visant initialement des objectifs d'efficience des modes de production, il s'est peu à peu modifié au début du XXI ${ }^{\text {ème }}$ siècle pour se focaliser sur les émissions de gaz à effet de serre. De nos jours, le facteur 4 , selon l'échelle d'étude à laquelle on se place, peut faire référence à deux ensembles différents mais reliés entre eux. Le premier facteur 4 dit "climatique", pour les pays industrialisés (échelle macro-économique), a été introduit pour limiter les émissions de gaz à effet de serre (GES) à venir au cours des prochaines décennies. Il consiste à diviser par 4 les émissions de gaz à effet de serre $\left(\right.$ teq $\left.\mathrm{CO}_{2}\right)$ des pays "développés " afin notamment de stabiliser au niveau mondial l'augmentation de température à $+2^{\circ} \mathrm{C}$. De fait, un tel engagement au niveau macro-économique ne peut être obtenu que par des actions ciblées sur les secteurs les plus émetteurs au niveau microéconomique. Ce constat s'est traduit par l'introduction d'un second concept : le facteur 4 « énergétique ». Le facteur 4 énergétique est une application au niveau local du facteur 4 climatique. De fait, les consommations d'énergie émettent des GES et leur limitation provoquerait en théorie une diminution proportionnelle de ces émissions. Le bâtiment en tant que consommateur d'énergie et émetteur important de GES est l'un des secteurs qui a connu le plus grand engouement, notamment par l'apparition de labels énergétiques et environnementaux. De fait, ces derniers ont su s'insérer sur un domaine encore vierge pour imposer leur vision et leurs objectifs. Ces objectifs se traduisent par une évaluation qualitative ou quantitative, selon des thématiques spécifiques (énergie) ou plus générales (environnement). Les labels existent parce que des maitres d'ouvrage considèrent que leurs enjeux « en valent la chandelle » et acceptent le surinvestissement induit afin de créer des "vitrines" du bâtiment de demain. De ce fait, les labels peuvent faciliter l'expérimentation de techniques innovantes et contribuer à l'amélioration de la performance des bâtiments. En cela, ils s'insèrent donc dans la direction du facteur 4 (énergétique et climatique) sans pour autant le garantir. Cependant, l'orientation des labels et leur coût, couplés à leur caractère volontariste, limite leur portée et leur reproductibilité à grande échelle. Les labels ne sont donc qu'une porte d'entrée, parmi d'autres, au facteur 4 , un guide à suivre plus ou moins scrupuleusement dans l'optique 
d'une démocratisation de la " performance » des bâtiments et ce aussi bien d'un point de vue énergétique, environnemental que social.

\section{BIBLIOGRAPHIE}

Abdelghani-Idrissi M. A., Birot J. J., Seguin D., Miller A., Ip K., 2004, Outils d'analyse environnementale des bâtiments, Réseau International de Développement Durable du Bâtiment, Durabuild

ADEME, 2007, Guides des facteurs d'émissions, ADEME, MIES

Association Effinergie, 2008, Réussir un projet de Bâtiment Basse Consommation, Guide à destination des professionnels du bâtiment, Association Effinergie

Association Minergie, 2008, Minergie-Eco, meilleure qualité de vie, respect de l'environnement Association négaWatt, 2006, Pour un avenir énergétique sobre, efficace et renouvelable, Scénario NégaWatt 2006, 1.0.2, Association négaWatt

Boutaud A., 2004, Le développement durable : penser le changement ou changer le pansement ? Thèse ENSM-SE

BRE, 2008, BRE Environmental \& Sustainability Standard, BREEAM Courts 2008 Assessor Manual, BES 5050, issue 2.0, BRE Global

Canada Green Building Council, 2004, LEED Green Building Rating System, Système d'évaluation des bâtiments écologiques pour nouvelles constructions et rénovations importants, LEED Canada-NC Version1.0, GBC

Certivéa, 2008, Référentiel du Système de Management de l'Opération, Référentiel du système de management de l'opération - Bâtiments tertiaires, Certivéa

CIAD (Comité interministériel pour le développement durable), 2008, Stratégie nationale de développement durable 2010-2013 - Pour une économie verte et équitable, disponible sur http:// www.developpement-durable.gouv.fr/IMG/pdf/SNDD_index.pdf

Ding G. K. C., 2008, "Sustainable construction. The role of environmental assessment tools", vol. 86, $\mathrm{n}^{\circ}$ 3, Journal of Environmental Management, p. 451-464

Eichholtz P., Kok N., Quigley J. M., 2009, Doing Well by Doing Good? Green Office Buildings

Envirobat, 2007, Analyse de la certification Maisons de Qualité, Envirobat

GIEC, 2001, Bilan 2001 des changements climatiques : Mesures d'atténuation, $3^{\mathrm{e}}$ rapport du GIEC

GIEC, 2007, Changements climatiques 2007. Rapport de synthèse, GIEC

Green Mark, 2008, BCA Green Mark for Residential Building, Version RB/3.0, BCA Green Mark

Grenelle Environnement - Groupe 1, 2008a, Lutter contre les changements climatiques et maîtriser l'énergie, Le Grenelle Environnement

Grenelle Environnement - Groupe 1, 2008b, Synthèse et principales mesures, Le Grenelle Environnement 
GT Facteur 4, 2006, Rapport du Groupe de Travail « Division par quatre des émissions de gaz à effet de serre de la France à l'horizon 2050 ", Ministère de l'Économie des Finances et de l'Industrie

Illich I., 1973, Énergie et équité, Edition le seuil, Paris

INSEE, 2009, http://www.insee.fr/fr/regions/bourgogne/, Consulté en mars 2009

JSBC, 2008, CASBEE for New Construction, IBEC

La maison passive France, 2007, Certification en tant que « Maison Passive certifiée », Certification en tant que « Maison Passive certifiée » Critères pour les maisons passives en tant que bâtiment d'habitation, La Maison Passive France.

Loi POPE, 2005, Loi $n^{\circ} 2005-781$ du 13 juillet 2005 de programme fixant les orientations de la politique énergétique

MECSL (Ministère de l'Emploi de la Cohésion Sociale et du Logement), 2007, Arrêté du 3 mai 2007 relatif au contenu et aux conditions d'attribution du label « haute performance énergétique "

MEEDDEM (Ministère de l'Écologie, de l'Énergie, du Développement Durable et de la Mer), 2007, http://www.statistiques.developpement-durable.gouv.fr, Consulté en novembre 2009

MIES, 2008, http://www.effet-de-serre.gouv.fr/, Consulté en novembre 2009

Nations Unies, 1998, Protocole de Kyoto de la à la convention-cadre des nations unies sur le changement climatiques, Nations Unies

NF ISO 15392, 2008, Développement durable dans la construction

Observatoire de l'énergie, 2009, http://www.industrie.gouv.fr/energie/sommaire.htm, Consulté en mars 2009

Partenay C., 2005, « Herbert Simon : rationalité limitée, théorie des organisations et sciences de l'artificiel », Groupe Réseaux Jean Monnet (GRJM), WP-2005-7, http://www.grjm.net/documents/ claude_parthenay/Parthenay_Simon.pdf, consulté en avril 2010

Population Reference Bureau, 2008, Fiche de données sur la population mondiale 2008, PRB.

Qualitel, 2008, Habitat \& Environnement, Millésime 2008, DER - groupe Qualitel

Schneider F., 2003, «L'effet Rebond ", L'Écologiste, édition française de The Ecologist, vol. 4, n 3, p. 45, http://www.decroissance.org/francois/recherche/articles/effet_rebond.pdf

SIA, 2009, Certificat énergétique des bâtiments selon SN EN 15217 et SN EN 15603, Société suisse des ingénieurs et des architectes

Sidler O., 2003, Diviser par 4 la consommation d'énergie des bâtiments : mythe ou réalité ? Enertech, http://www.prohabitat.ch/upload/ecohabitat/editor/pdf/diviser_par_4 \%20la \% 20consommation \%20denergie.pdf

Sidler O., 2007, Rénovation à basse consommation d'énergie des logements en France, Enertech, Projet « RENAISSANCE », Programme européen CONCERTO, Enertech, http://www.negawatt.org/ telechargement/Docs/Sidler \%20Renovation \%20final \%201107.pdf

Simon H. A., 1947, Administrative Behavior. A study of Decision-Making Processes in Administrative Organization, traduction française par Pierre-Emmanuel Dauzat, 1983, Economica, Paris

Simon H. A., 1992, « De la rationalité substantive à la rationalité procédurale », disponible sur le site MCX - APC, http://www.mcxapc.org/docs/lesintrouvables/simon5.htm, issu de la revue PISTES, $n^{\circ}$ 3, octobre 
Taylor F., 1911, The Principles of Scientific Management, Harper \& Brothers, New York, NY, USA and London, UK, http://www.gutenberg.org/ebooks/6435

Tribu Énergie, 2006, La méthode de Calcul des Consommations Conventionnelles dans les Logements, Tribu Énergie

Tuddenham M., 2006, Historique du Facteur 4, CITEPA

Villot J., Gondran N., Laforest V., 2009, La vision de la construction « durable » au regard des labels, Rapport IFPEB

Von Weizsäcker E. U., Lovins A. B., Lovins L. H., 1997, Facteur 4 : deux fois plus de bien-être en consommant deux fois moins de ressources - Rapport au Club de Rome, Terre Vivante, Mens

\section{RÉSUMÉS}

Le facteur 4, souvent évoqué comme objectif à atteindre face aux enjeux énergétiques et au changement climatique, est caractérisé par une double identité. Faisant à la fois référence à une augmentation de la productivité des ressources (facteur 4 " énergétique ») et à l'atténuation des émissions de gaz à effet de serre (facteur 4 « climatique »), il constitue l'objectif à atteindre dans les 40 ans. Dans ce but, différents secteurs d'activité se sont approprié ces concepts. Dans le secteur de la construction, la volonté d'améliorer les performances énergétiques et environnementales des bâtiments est passée par la création depuis les années 1990 des systèmes de labellisation. Les labels, par leurs approches normatives, se veulent être une des voies permettant de mettre en place les changements nécessaires pour atteindre les facteurs 4 . Le but de cet article est d'étudier les différents types de labels de la construction et de voir comment et jusqu'à quel point ils permettent, ou non, d'atteindre les objectifs des facteurs 4.

Factor 4, which is often presented as an objective to deal with energy and climate change issues, is characterized by a double identity. Referring at the same time to an increase of resources productivity (factor 4 for energy) and to greenhouse gas attenuation (factor 4 for climate), it constitutes challenge to be reached in 40 years. To this end, the various branches of industry have adapted these concepts. In the building sector, this appropriation has been translated since 1990 by the creation of labeling systems. The labels by their standardized approach endeavor to be the precursors and the essential to drive these changes to the factors 4 . The goal of this paper is to study the various types of labels that apply to sustainable construction and to study how and at which degree they contribute to factors 4 objectives.

\section{INDEX}

Mots-clés : facteur 4 climatique, facteur 4 énergétique, labels de la construction

Keywords : factor 4 for climate, factor 4 for energy, labeling systems

\section{AUTEURS}

\section{JONATHAN VILLOT}

Jonathan Villot est doctorant à l'École Nationale Supérieure des Mines de Saint-Etienne. ENS des Mines de Saint-Etienne, Sciences, Information et Technologies pour l'Environnement 158, cours Fauriel 42023 Saint Étienne cedex 2 villot@emse.fr 


\section{NATACHA GONDRAN}

Natacha Gondran est enseignante-chercheur au centre SITE (Sciences, information et technologies pour l'environnement) de l'École Nationale Supérieure des Mines de Saint-Étienne. ENS des Mines de Saint-Etienne, Sciences, Information et Technologies pour l'Environnement 158, cours Fauriel 42023 Saint Étienne cedex 2

\section{VALÉRIE LAFOREST}

Valérie Laforest est enseignante-chercheur au centre SITE (Sciences, information et technologies pour l'environnement) de l'École Nationale Supérieure des Mines de Saint-Étienne. ENS des Mines de Saint-Etienne, Sciences, Information et Technologies pour l'Environnement158, cours Fauriel42023 Saint Étienne cedex 2 